\title{
Vector Bessel Beams with Modified Field Distribution
}

\author{
Sergej Orlov, Ada Gajauskaitė and Artūras Grabusovas \\ Center for Physical Sciences and Technology, Industrial laboratory for photonic technologies, \\ Sauletekio av. 3, LT-10222, Vilnius, Lithuania \\ E-mail: sergejus.orlovas@ftmc.lt
}

\begin{abstract}
In this work we theoretically introduce and experimentally examine a novel type Bessel-like beams which have an intensity distribution spherically modified by a polarization structure when compared to standard Bessel beams. Standard Bessel-Gaussian beams can be either linearly or radially and azimuthally polarized. Here, the Bessel-like beams with a Gaussian envelope, we are introducing, can have either spherically radial or meridional - azimuthal polarizations. In order to implement those beams experimentally we investigate their vector spatial spectra numerically for the case of a finite energy. A proper choice of the cone angles of individual plane wave components and sizes of Gaussian apertures, we can control experimentally intensity distribution of resulting beams. Lastly, we demonstrate that in two limiting cases we can form from our solutions either two optical needles or doughnut-like intensity patterns.
\end{abstract}

DOI: 10.2961/jlmn.2018.03.0013

Keywords: nondiffracting beams, diffraction, polarization, beam shaping, Bessel beam.

\section{Introduction}

An interest in laser beam shaping and exotic beam formation was on a rise in the recent years in order to satisfy either some specific practical applications or as well as a general pure scientific interest. A good example of structured light is a nondiffracting Bessel-Gauss beam which exhibits a long focal line and is well known for its selfreconstructing properties [1,2]. In a similar fashion, additional degree of freedom in the transvers profiles with asymmetrical intensity distributions, which have comparable to Bessel beam properties, can be achieved after introducing the so-called Mathieu-Gaussian and parabolicGaussian (Weber-Gaussian) beams. [3,4].

Usually Bessel beams are considered as scalar beams because their angular frequencies are low. However, when the angle of the Bessel cone is large, scalar description is not valid anymore and one needs to introduce a vector one. Within the vector description a Bessel beam becomes nonhomogeneously polarized, and even the longitudinal component of the electric field can appear. A good example of this situation are radially polarized beams, where tight focusing can further increase the longitudinal component of the electric field of the beam as plane wave components with nearly perpendicular to the propagation axis angles are impinging upon the focal spot. Interestingly, this situation can lead to improved laser microfabrication rates in hole drilling operations as it was experimentally confirmed for azimuthally and radially polarized Bessel beams recently [5].

The influence of the nonhomogeneos polarization state of the beam on its structure usually results in polarization singularities, which are present in the vector beams. They distort the beam profile by the introduction of an intensity zero, which, as a rule, is cylindrically symmetric both for azimuthally and radially polarized beams.

However, within the electromagnetics, there are so called vector spherical multipoles, which can have two interesting polarization states, which can be called spherically radial (electric multipoles) or meridional - azimuthal (magnetic multipoles). Those nonhomogeneous polarizations exhibit some intriguing properties: a) they are spherically symmetric and b) they have a so-called doughnut shape.

So called optical doughnut beams are considered as promising for optical manipulation systems, where structured optical fields are employed to handle small objects. These beams have zero intensity region at the center of the beam [6].

In this work we investigate a Bessel like beam, which has a nonhomogeneous polarization state, which can be classified as meridional - azimuthal. We consider here this novel type of a beam for two cases with two distinct types of intensity distributions that display appearance of an optical doughnut, mentioned already, and of a structure, which can be called a double needle.

\section{Theory}

Optical beams that can be mathematically expressed using separable variables both in the transverse and longitudinal planes and which are solutions of Helmholtz equation usually are called nondiffracting beams. A Bessel beam is a good example, which describes a beam in the circular cylindrical coordinates. Its complex amplitude can be written as

$A(\rho, \varphi)=J_{m}\left(k_{\rho_{0}} \rho\right) \exp (\mathrm{i} m \varphi)$

where $J_{m}$ is $m$-th order Bessel function of the first kind, $\rho, \varphi$ are polar coordinates, $k_{\rho_{0}}=k \sin \theta$ and $k_{z}=k \cos \theta$ are transverse and longitudinal components of the wave vector $\mathbf{k}, \theta$ - is a half angle of the Bessel cone, $m$ - is a topological number (or a topological charge), that describes phase front dislocation (an optical vortex present in the 
wavefront of a higher order Bessel beam). A spatial spectrum of a Bessel beam can be written as:

$s_{B}\left(k_{\rho}, \phi\right)=\frac{1}{2 \pi k_{\rho}} \delta\left(k_{\rho_{0}}-k_{\rho}\right) \exp (\mathrm{im} \phi)$,

where $\delta$ is a delta function and $\phi$ is an azimuth angle of a spatial spectrum.

An ideal Bessel beam is endless in the space and has an infinite amount of the energy. Experimentally obtainable beams (also known as Bessel-Gaussian beams) are bound in space and have a finite amount of the electromagnetic energy. For the Bessel-Gaussian beam a new concept of the Bessel zone arises. This is the zone, where the BesselGaussian beam exists. The length of the Bessel zone $z_{B}$ depends on the angle of the Bessel half-cone and the width of its Gaussian aperture $d_{0}$ and can be expressed as $z_{B}=d_{0} / \tan \theta$. Traditionally, experimentally realizable Bessel beams can be obtained using a combination of a narrow ring type aperture and a Fourier lens. A more efficient way to produce a Bessel beam is to use a conical prism (an axicon), which modulates only the phase of incoming beam. An example of a Bessel-Gaussiam beam is depicted in the Fig. 1.
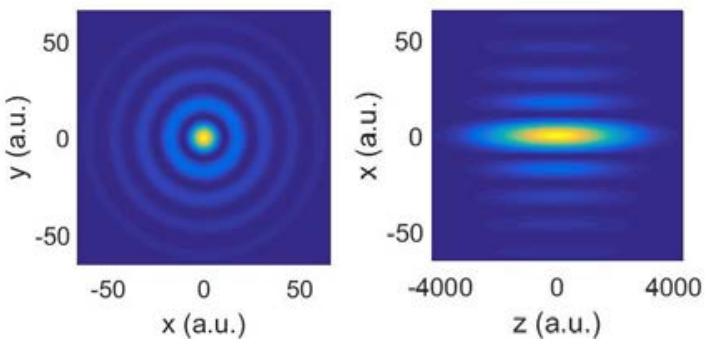

Fig. 1 Intensity distribution of a scalar Bessel-Gauss beam in the transverse (a) and longitudinal (b) planes for $m=0$. All units are normalized to the wavelength of the beam.

The complex amplitude of a vector beam can be expressed as a superposition [7-9]:

$\boldsymbol{E}(\boldsymbol{r})=\sum_{n} c_{n} \boldsymbol{M}_{n}(\boldsymbol{r})+f_{n} \boldsymbol{N}_{n}(\boldsymbol{r})$,

where $c_{n}$ and $f_{n}$ are coefficients and $\boldsymbol{M}, \boldsymbol{N}$ - are vector fields, which were derived from a scalar solution of the scalar Helmholtz equation using the following approach from the Ref. [8]:

$$
\begin{aligned}
& \boldsymbol{M}(\boldsymbol{r})=\nabla \times[\boldsymbol{a} A(\boldsymbol{r})], \\
& \boldsymbol{N}(\boldsymbol{r})=1 / k[\nabla \times \boldsymbol{M}(\boldsymbol{r})],
\end{aligned}
$$

where $\boldsymbol{a}$ is a some vector. In this work, we construct a vector Bessel beam when $\boldsymbol{a}$ is a radius vector $\mathbf{R}$ of the spherical coordinate system:
$\boldsymbol{a}=\mathbf{R}=x \boldsymbol{e}_{x}+y \boldsymbol{e}_{y}+z \boldsymbol{e}_{z}$,

where $x, y$ and $z$ are Carthesian coordinates and $\boldsymbol{e}_{x}, \boldsymbol{e}_{y}$ and $\boldsymbol{e}_{z}$ are unit vectors of the coordinate system.

As it was demonstrated by Stratton [8], any vector solutions obtained using this vector do satisfy the boundary conditions on a sphere. Thus, for our purpose it would be interesting to investigate how the scalar Bessel beam will be transformed during this vectorization approach.

In general, two main modes, which are described by vector fields $\boldsymbol{M}$ and $\boldsymbol{N}$, have physical meaning and can be observed in the free space. The electric field in the TE mode is tangential to the surface of the sphere (vector field $\boldsymbol{M}$ ) and the electric field in the TM mode is pointing normally to the surface of the sphere (vector field $\boldsymbol{N}$ ).

In this work we study a specific case when $f_{n}=0$, see Eq. (3). Thus, expressions for the components of an ideal Bessel beam in our case are as follows:

$$
\begin{aligned}
& M_{\rho}(\rho, \varphi, z)=\mathrm{i} \frac{m z}{\rho} J_{m}\left(k_{\rho_{0}} \rho\right) \exp \left(\mathrm{i} k_{z} z+\mathrm{i} m \varphi\right) \\
& M_{\varphi}(\rho, \varphi, z)=\left[\mathrm{i} k_{z} \rho J_{m}\left(k_{\rho_{0}} \rho\right)-z J_{m}^{\prime}\left(k_{\rho_{0}} \rho\right)\right] \times \\
& \exp \left(\mathrm{i} k_{z} z+\mathrm{i} m \varphi\right) \\
& M_{z}(\rho, \varphi, z)=-\mathrm{i} m J_{m}\left(k_{\rho_{0}} \rho\right) \exp \left(\mathrm{i} k_{z} z+\mathrm{i} m \varphi\right)
\end{aligned}
$$

Due to the space limitations we omit the expressions for the components of a vector Bessel-Gauss beam. They will be presented elsewhere, however we will demonstrate later the properties of those vector beams.

We start with an example of a numerically calculated transverse intensity distribution of a Bessel-Gaussian type optical beam for two different on-axis distances $z$, results are shown in the Fig. 2.
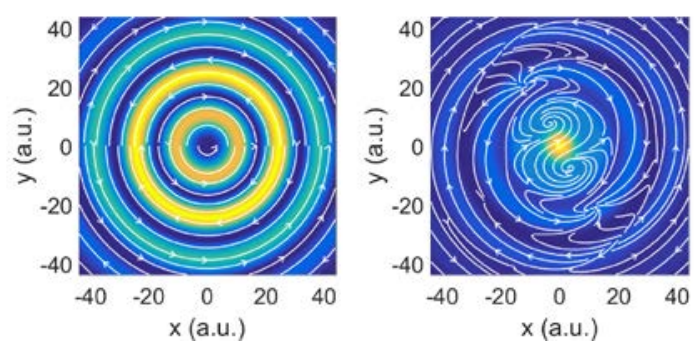

Fig. 2 Intensity distributions of meridional - azimuthal polarized Bessel-Gaussian beams $|\boldsymbol{M}|^{2}$ in the transverse $(x, y)$ plane for $d_{0}=40$. Cross sections were computed at (left) $z=0$ and (right) $z=1$ distances. Lines here depict directions of the electric field.

We obtain the vector spatial spectra of these vector Bessel beams by numerically performing the Fourier transform for the individual components the beam. 


\subsection{Experimental setup}

The experimental setup for generation of the vector components of the spatial spectrum is presented in the Figure 3 . The $4 f$ lens system is used to transfer the spectral image from the SLM to a lens (L4). Here, after the Fourier transform, we generate the desired beam profile which is captured and recorded afterwards by the imaging system. Parameters of experimental setup are: $100 \mathrm{~mW}, 532 \mathrm{~nm}$ CW DPSS laser (Changchun New Industries Optoelectronics Technology Co., Ltd.), HWP - half wave retarder plate, POL -Brewster polarizer, ND - neutral density attenuating filter, OBJ1 -objective lens, L1 -collimating lens, M1 mirror, AP1 -aperture, BS - beam splitter, SLM - phaseonly spatial light modulator (PLUTOVIS-006-A, HOLOEYE Photonics AG), L2-L3 -lenses in 4f configuration, L4 - Fourier transform lens, imaging optics OBJ2-L5 and CCD camera are set up on a motorized translation stage.

A complex-valued spatial spectrum of a single vector component is converted to a phase-only spatial phase-mask using the so-called checkerboard method.

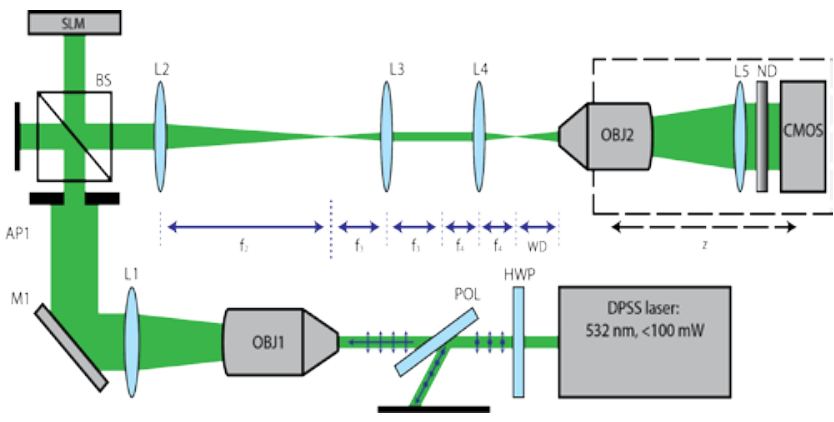

Fig. 3 Sketch of the experimental setup. CW laser, lenses (L1-L5), objectives (OBJ1 and OBJ2), spatial light modulator (PLUTOVIS-006-A, HOLOEYE Photonics AG) and a CCD camera

\section{Experimental and numerical results}

Numerically calculated vector components of a spatial spectrum are depicted in the Fig 4. As one can see, the spatial spectrum consists of two rings with angles $\theta_{1}=3.4^{\circ}$ and $\theta_{1}=4.6^{\circ}$, where each individual ring has its own phase modulation.
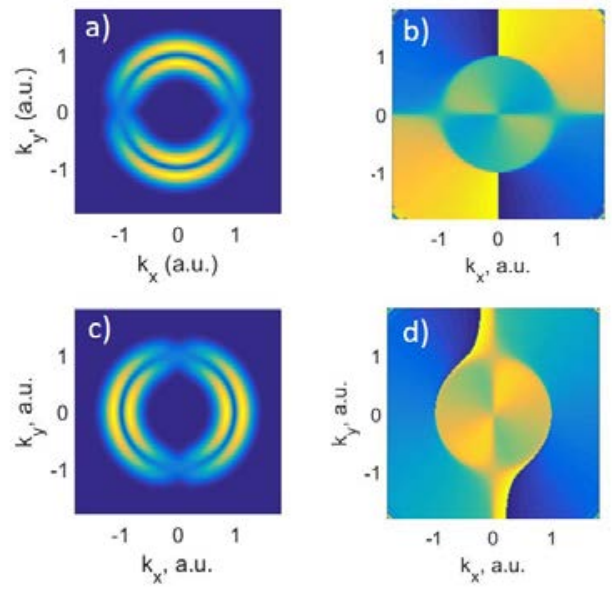

Fig. 4 Intensity (left) and phase (right) distributions of the vector components of the spatial spectrum, when $m=1$ and $d_{0}=12$. The first line is the $x$ component, and second - the $y$ component.
Vector components of the spatial spectrum were numerically calculated and after encoding into the phase-only masks were uploaded onto the matrix of the SLM. The outcome of this operation are experimentally measured array of 2D intensity distributions for each individual vector components.

An example of such experimental measurement is depicted in the Fig. 5, For the sake of brevity only a single case of a $x$ component of the beam is presented. As we observe, the linear focus consists now not from a single line as in the case of the classical Bessel Gaussian beam but it has two distinct zones of the non-zero intensity. Those non-zero zones are separated by a zone with nearly zero intensity at the very center of the optical beam. In the $(z, y)$ plane we can observe the distinct ring structure of a Bessel beam, while in the $(z, x)$ plane only line light pattern can be seen. The $y$ component of the beam is rotated by the angle of $\pi / 2$ in the $(x, y)$ plane when compared to the $x$ component, therefore its properties are similar, what justifies our decision to omit its graphs here.
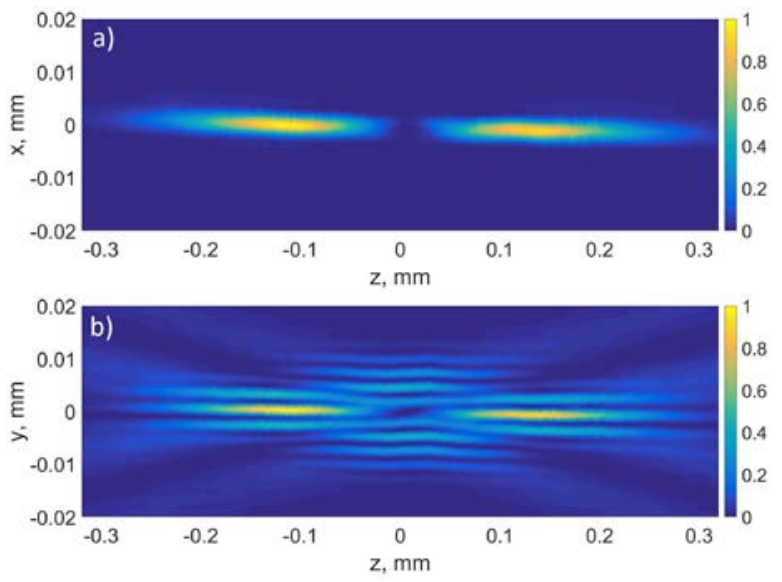

Fig. 5 Experimentally obtained intensity distribution of the $E_{x}$ component of a meridional - azimuthal polarized

Bessel-Gaussian beam in two planes a) $(z, x)$ and $b)$

$(z, y)$. Topological charge $m=1$ and aperture $d_{0}=12$.

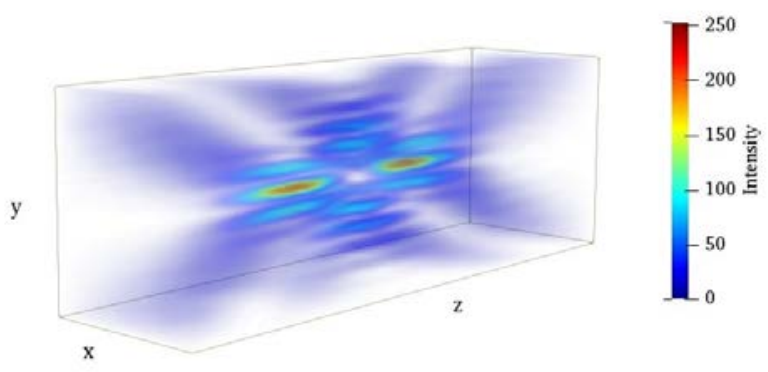

Fig. 6 A three-dimensional depiction of the experimentally obtained intensity distribution of the $E_{x}$ component of

the meridional - azimuthal polarized Bessel-Gaussian beam. For better visualization of the field the opacity is set at small values of the intensity. Topological charge $m=1$ and Gaussian aperture $d_{0}=12$. 
A 3D intensity distribution of the $x$ component is presented in the Fig. 6. As we observe the round Bessel-like rings, which are azimuthally polarized, are formed only at the beam waist or in the very center of the Bessel zone. Moreover, as we see, the intensity on the optical axis increases when going further from the center of the beam.

This is the best manifestation of the changes in the intensity distribution, which are extensively caused only by the polarization structure of the beam. As the beam propagates, we can observe its structure at each plane and we can see that the polarization changes during the propagation. At the very center of the beam it forms a structure, which can be described as a circularly polarized state, a rotating polarization state. A bit further away from the center of the beam, at the periphery the polarization state can be described in classical terms as azimuthal.

Thus, both complicated polarization structure and intensity distribution can be observed for this particular case. This especially good can be seen by individually inspecting the individual beam components at different propagation distances.

Transverse intensity distributions of the $x$ component at different distances from beam waist are depicted in the Fig. 7. Results of our numerical modeling (Fig. 7 (a-c)) perfectly coincide with our experimental results here, see Fig. 7 (d-f). While propagating, the transverse field experiences rotation and its intensity is localized at the center of the beam.

A naïve inspection of Eqs. $(7,8)$ reveals, that for $z=0 \mathrm{~mm}$, the meridional - azimuthal polarized Bessel-Gaussian beam is described as a superposition of the $m$-th order Bessel function, and for $z \square 0$, or for situations outside the center of the beam, the beam is described as a sum of Bessel with topological numbers $m-1$ and $m+1$. Therefore a rotation of the intensity pattern is observed, see Fig 7.
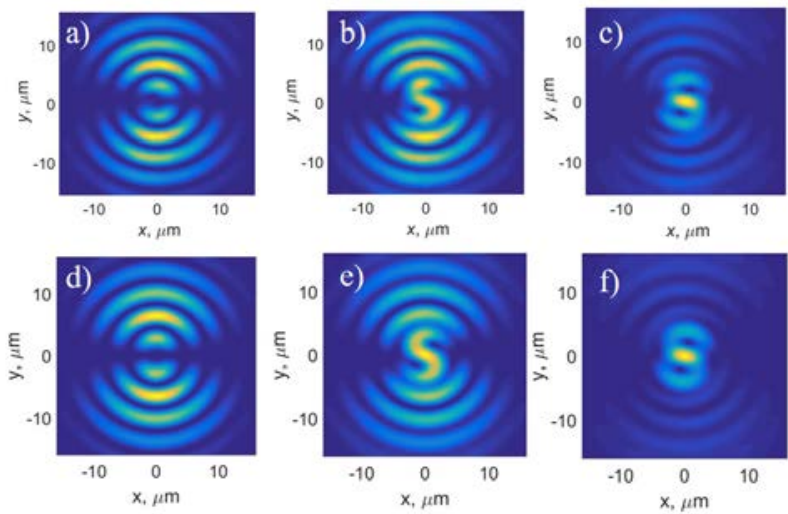

Fig. 7 Intensity distribution in the transverse plane for two cases of experimentally observed (a-c) and numerically calculated (d-f) vector component $E_{x}$ of the meridional azimuthal polarized Bessel-Gaussian beam with $d_{0}=7$ at following distances: (a,d) $z=0 \mathrm{~mm}$, (b,e)

$z=0.05 \mathrm{~mm},(\mathrm{c}, \mathrm{f}) \mathrm{z}=0.1 \mathrm{~mm}$ from the focal plane.

In another limiting case, when the aperture of the Gaussian envelope is small (i.e. the ring in spatial spectrum is rather wide), the physical properties of the Gaussian beam are dominant in the meridional - azimuthal polarized BesselGaussian beam. As an example of this situation, we will now examine this limiting case. Here, the parameter $d_{0}$ has to be small enough, to not observe the Bessel function in the intensity pattern. An experimental example of this situation is presented in the Figure 8.
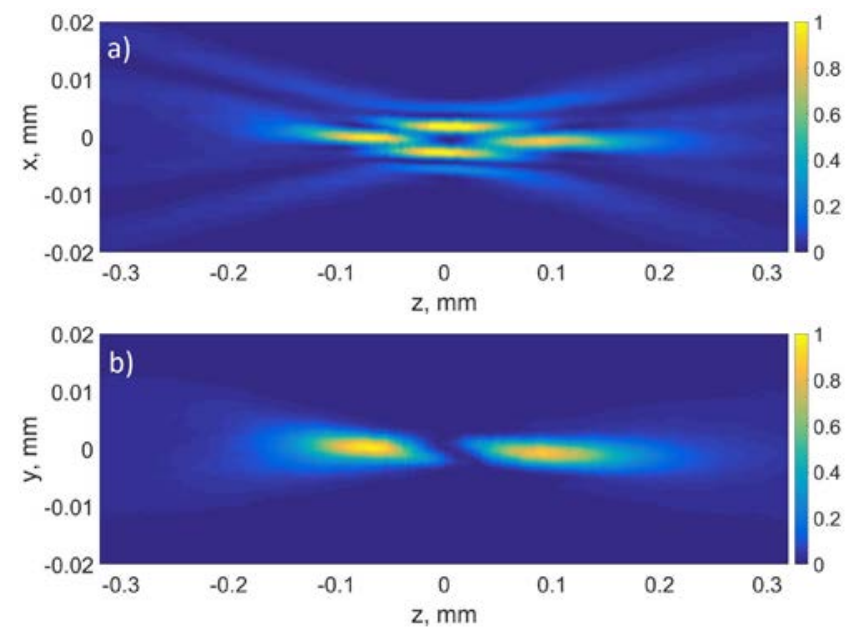

Fig. 8 Experimentally obtained intensity distribution of the $E_{y}$ component of the meridional - azimuthal polarized Bessel-Gaussian beam, for a particular case, when the Gaussian envelope is rather small.

Here, two different cross-sections of the intensity of the $E_{y}$ component of the the meridional - azimuthal polarized Bessel-Gaussian beam are investigated. We note that the intensities of both ( $x$ and $y$ ) components form doughnutlike intensity patterns, see Fig. 8. A three-dimensional visualization of two individual components for this particular case are depicted in the Fig. 9.

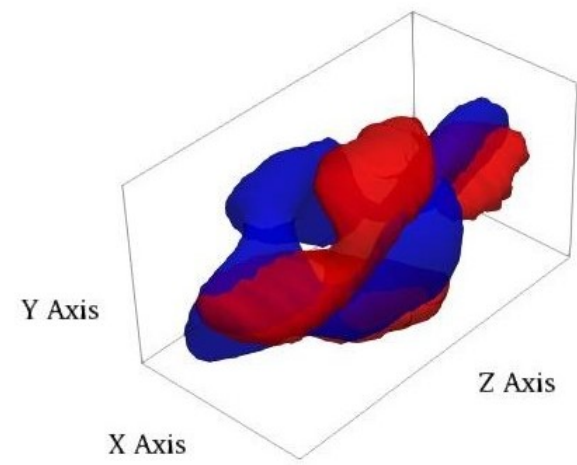

Fig. 9 A depiction of two experimentally obtained isosurfaces: the $E_{x}$ component (red) and $E_{y}$ component (blue) of the meridional - azimuthal polarized BesselGaussian beam for small ( $d_{0}=7$ ) values of Gaussian anortiors

Thus, a proper choice of the angles of the Bessel cone and sizes of Gaussian envelopes, it is possible to control the intensity distribution of resulting beams. It seems, that this intensity control has two rather different limiting cases encoded as its distinct features.

\section{Conclusions}

In this work we have both theoretically, numerically and experimentally examined a novel type of Bessel-like beam, 
which have a novel spatial structure due to meridionalazimuthal polarization state, which has drastically modified the standard intensity profile of a common Bessel beam.

Nonhomogeneous polarizations like azimuthal and radial are becoming important in areas of laser microprocessing, as the number of reports on their efficiency for material processing applications is increasing $[10,11]$. Given their unique properties and nondiffracting properties combined with spherical polarization nature meridional - azimuthal polarized Bessel-Gaussian beams should be interesting for laser microprocessing applications, when round cuts and controllable corners are important.

\section{Acknowledgments}

This research is funded by the European Social Fund according to the activity 'Improvement of researchers' qualification by implementing world-class R\&D projects' of Measure No. 09.3.3-LMT-K-712.

\section{References}

[1] J. Durnin: J. Opt. Soc. Am. A, 4, (1987) 651.

[2] F. Gori, G. Guattari, and C. Padovani: Opt. Commun., 64, (1987) 491.

[3] J.C. Gutiérrez-Vega, and M. A Bandres: J. Opt. Soc. Am. A., 22, (2005) 289.

[4] M.A. Bandres, J.C. Gutiérrez-Vega, and S. ChávezCerda: Opt. Lett., 29, (2004) 44.

[5] R. Drevinskas, J. Zhang, M. Beresna, M. Gecevičius, A.G. Kazanskii, Y.P. Svirko, and P. G. Kazansky: Appl. Phys. Lett., 108, (2016) 221107.

[6] L. Gong, W. Liu, Q. Zhao, Y. Ren, X. Qiu, M. Zhong, Y. Li: Sci. Rep., 6, (2016) 29001.

[7] Z. Bouchal, and M. Olivík: J. Mod. Opt., 42, (1995) 1555.

[8] J.A. Stratton. "Electromagnetic theory" (John Wiley \& Sons; 2007).

[9] P.M. Morse, and H. Feshbach. "Methods of theoretical physics" (Mc-Graw Hill; 1953).

[10]M. Meier, V. Romano, and T. Feurer: Appl. Phys. A, 86 (2007) 329.

[11]M. Kraus, M. A. Ahmed, A. Michalowski, A. Voss, R. Weber, and T. Graf: Opt. Express, 18, (2010) 22305.

(Received: July 26, 2018, Accepted: October 13, 2018) 